\title{
A New System Framework of University Campus Information Navigation Based on Android Platform
}

\author{
J.Y.LI \& D.WANG \& L.N FAN\&S.K.SHI \& N.W.YAO \\ Department of Information Engineering, Shenyang University, Shenyang 110044, China \\ Department of Information Engineering, Shenyang University, Shenyang 110044, China
}

\begin{abstract}
KEYWORD. Android Platform; System Framework;Campus Navigation Platform;Digital Campus ABSTRACT. Digital campus is an important part of the University's information.More and more students have mobile phones, so mobile phones have become an important part of the students.Mobile applications to help people to do some things and make lives become more convenient.In this paper, we design a new comprehensive information platform to meet the needs of students.The main function of this integrated information platform is to make the learning and life more convenient and efficient.
\end{abstract}

\section{INTRODUCTION}

Now the world has entered into 21 st century, in which information develops fast.In the economic and social activities, obtaining valid information can save time, and achieving goals more quickly. Information has become an important strategic resource during the process of the development of a country and a region.[1] "Digital Campus" has become an important strategic target of many colleges and universities.Developing a college campus counseling platform based on GPS, GIS and MapX will become a crucial part of campus informationalized strategy[2].

\section{SYSTEM REQUIREMENTS ANALYSIS}

School is a dense place with many people and buildings.A lot of people in the school can not find their way[3].They need to ask for directions sometimes, sometimes the people whom they asked do not know the way either, in this situation it will delay people's time, they will even miss the best time to do things.

At the same time, many colleges and universities are used to the examination room in the weekend.Most of the people come outside the school.So they don't know the geographical location of examination room and teaching building. Then,it will delay the time and affect the attitudes of the test.

Since the current universities generally have multiple campuses, the students will be in different campuses[4].The signs in campus road are not very complete and clear, resulting in a lot of students do not know the location of each building,and get lost. After preliminary investigation and research, it is found that the electronic map will bring convenience to people.

The initial target market of this platform is for the students in school and off campus personnel, through the electronic map navigation ,they can complete their own destination that they want to go to the with the shortest path. With the in-depth research, we team found that we can embed this APP product into school information consulting module and abundant the function.so that it can provide good information for more internal and external users.

\section{ANDROID ARCHITECTURE AND FEATURES}

The important characteristics of the Android system

The development of this platform is mainly based on the Android system, because the Android system has the important characteristics of the following aspects. 
openness

The development of Android platform allows any mobile terminal manufacturers to join the Android alliance. Significant openness can make it have more developers, with the increasing use of users and applications, a new platform will become mature very soon.

\section{Not Bound By the Operator}

Since Android listed, the user can conveniently connect to the network and reduce the restriction of operator. With gradual transition and enhance edge, HSDPA these $2 \mathrm{G}$ to $3 \mathrm{G}$ mobile network, mobile freely to network has not operators longer joke.

A Wealth of Hardware Options

Due to the openness of Android, many manufacturers will launch a variety of rich and colorful and distinctive products. Functional differences and characteristics will not affect the data synchronization, and even the compatibility of the software.

Without Any Restrictions On The Developer

Android platform can provide a broad and free environment for the third-party developers .Therefore are not subject to various rules of the obstruction.Imagining that this will be born many novel and unique software .

Seamless Combination Of Google Applications

Now Google in the Internet field has gone through 10 years of history, from the Search Giant to comprehensive Internet penetration, Google services such as maps, email, search,etc. and it has become an important connection between Internet users , and Android mobile phone platform will seamlessly the Google Apps.

\section{The Architecture of Android Platform}

Android system is designed based on the Linux kernel, and compare with Windows, Phone IOS and other operating systems, the fault tolerance ability is stronger. As an open source mobile device platform, and the system uses a layered architecture, the software hierarchy includes the operating system, UI layer, middleware and applications. The software hierarchy from top to bottom respectively Application, the Application Framework, Libraries, and the Android Run Time environment, the Operating System layer (OS) --Linux kernel . The following figure is the overall architecture of the Android operating system.As Figure 1.

Android is divided into four layers, from the top to the low layer is the application layer, application framework layer, the system runs the library layer and the Linux core layer. Blue on behalf of the $\mathbf{J}$ ava program, yellow code for running JAVA program to achieve the virtual machine, the green part is the $\mathrm{C} / \mathrm{C}$ language program library, red code kernel (Linux kernel driver). Under Framework Appl ication, by the C/C program library, through the JNI to complete the call from JAVA to C. APPLICATIONS

All applications are written in the Java language. Each application consists of one or more activities, activities must be to an activity to the super class.It is similar to the activities of the operating system process, but in contrast to the operating system process ,the activities are more flexible.'It is similar to the process, the activities can switch in a variety of states.

Using JAVA property of crossing platform, based on the development of the framework of the Android framework can not be compiled to run on any platform with a Android system, which is the essence of Android.

\section{LIBRARIES}

Application of architecture design simplifies the component reuse; any application can be released it' $s$ function block and any other applications can use the function block of the issue. (But it must follow the framework of security restrictions). Helping programmers to quickly develop the program, and the reuse mechanism of the application also allows users to easily replace the program components.

ANDROID RUNTIME

Android contains some $\mathrm{C} / \mathrm{C}$ libraries. the libraries can be used for different components in a Android system. They provide services to developers through the Android application framework. 
Mainly including the basic $\mathrm{C}$ library, as well as the multimedia library to support a variety of multimedia formats, bitmap and vector fonts, 2D and 3D graphics engine, browser, database support.

In addition,there is a hardware abstraction layer. In fact, Android is not all device drivers which are placed inside the Linux kernel.A part of the implementation are in the user space.

\section{LINUX KERNEL}

The core system services of Android rely on the Linux 2.6 kernel. It can build various drivers for the Android platform . enhancing the main effect of the overall terminal equipment and the quality of the core operation and effect[5].

\begin{tabular}{|c|c|c|c|}
\hline \multicolumn{4}{|c|}{ APPLICATIONS } \\
\hline Home & Dialer & Contacts & Browse \\
\hline \multicolumn{2}{|c|}{ LIVRARIES } & \multicolumn{2}{|c|}{ ANDROID } \\
\hline \multicolumn{2}{|c|}{ Media Framework } & & \\
\hline \multirow{2}{*}{\multicolumn{2}{|c|}{ Surface Manager }} & \multicolumn{2}{|c|}{ CoreJava Libraries } \\
\hline & & \multirow{2}{*}{$\begin{array}{l}\text { Dalvik } \\
\text { Machin }\end{array}$} & \multirow{2}{*}{ Virtual } \\
\hline & & & \\
\hline \multicolumn{4}{|c|}{ LINUX KERNEL } \\
\hline
\end{tabular}

Figure 1. The Base Architecture of Android System

\section{SYSTEM FRAMEWORK}

\section{Research of Market-determining}

School is a dense place, , which flows many people and building. A lot of people in the school can not find the place where you want to go.They need to ask for directions at any time, sometimes the people they ask do not know the way, in this situation it will delay people's time even lead the people miss the best time to do things.

At the same time, many colleges and universities are used to the examination room in the weekend.Most of the people come out of the school.So they don' $t$ know the geographical location of examination room and teaching building. In my experience, most of people come to our school to attend the examination $.80 \%$ of people don' $t$ know where is the room. Then, it will delay the time and affect the attitudes about the examination.

Since the current universities generally have multiple campuses, the students will be in different campuses.The signs in campus road are not very complete and clear, resulting in a lot of students do not know the location of each building,and get lost. After preliminary investigation and research, it is found that the electronic map will bring convenience to people.

Along with the rapid development of electronic information technology and the Internet, smart phones become indispensable part in people's lives, broadband, campus internet, 4G service extend to campus.they provided a running platform for campus navigation.

The platform's initial target market is the the University. The target people are in school students and off school staffs, they can complete their own destination that they want to go to the with the shortest path through electronic map navigation. 


\section{The System structure of this APP}

The platform is mainly for the teachers and students of the University, as well as new students.It can provide the school's electronic map and facilitate people's travel in the campus.

The people who need to use the platform need to download the app, in this app, they can look at the map of the campus and understand the layout of school, finding where to go.According to the destination,the system will locate the position,the system will provide the shortest path to the destination for customers.

\section{Studying the Main Functions of this APP}

Development of the University campus navigation based on intelligent mobile phone provide a campus navigation based on the Android platform for the university students, masters and off school staffs. We just need to install a smart phone APP, it allows you to easily locate their location, to find their own distance from the destination. This APP can be able to benefit the majority of teachers and students, in particular, the University freshmen that has just entered.

Campus navigation system is divided into the following functions.

(1) To look over the digital map of the University.

(2) To query the location of the administrative department.

(3) To query building details.

(4) To find all paths between the two buildings and the shortest path.

(5)To query path information.

(6) To find the joint points on the map.

(7) To look up the scenic spots on the map.

(8)To query the time to reach the destination

Research and development of the main modules of the campus information platform

The main module of the project's campus APP is to provide a campus information consultation platform based on Android platform for campus teachers, students and off school staffs . E-campus transportation -tiny campus treasure mainly includes campus navigation functions, the campus information platform, and various social examination room information. To achieve specific functions as follows.

(1) The functions of the campus navigation. browse digital map of colleges and universities, query part of the administrative location, query building details.to find all paths between the two buildings and the shortest path.to query path information.

to find the joint points on the map.to look up the scenic spots on the map.to query the time to reach the destination .

(2) Campus information platform. query library books, query curriculum, inquiry using classroom information, student achievement GPA statistics, campus recruitment information platform, the market of the graduated students .

(3) All kinds of social examination information. query the upcoming examination distribution map, search the examination location on each floor of the room layout. 


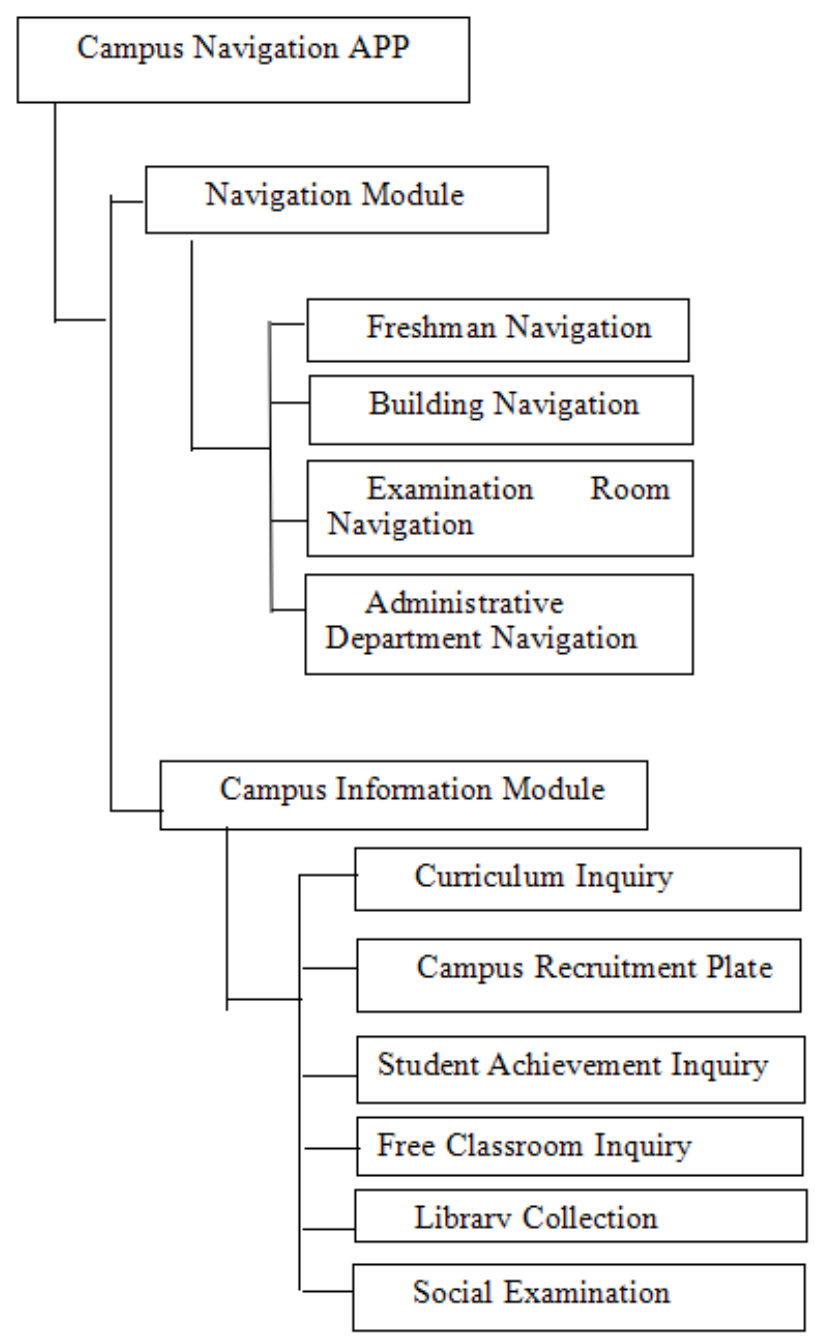

Figure 2. Function Module Diagram of This APP

\section{SUMMARY}

Campus information platform is an important part of a higher education institutions for the wisdom of the campus. Through the result of the research about the real need of in school students.Combined with the intelligent mobile terminal Android system, We come up with a conception of campus counseling integrated platform system design in this paper.

The integrated consulting platform will be integrated into the campus navigation, educational administration system, life information, employment information and students in all aspects of learning and life together.It will have realistic significance and value.

\section{REFERENCE}

[1]M.Q Song, H.Y Song, X.L Fu,

Methodology of user interfaces design based on Android, International Conference on Multimedia Technology (ICMT), 2011

[2]Y.H Cheng, W.K Kuo, S.L Su, An Android system design and implementation for Telematics services, Intelligent Computing and Intelligent Systems (ICIS), 2010

[3]M.Zhang, Research and Development of Virtual Digital Campus System Based on Android Platform,Intelligent Computation Technology and Automation (ICICTA), 2014 7th International Conference on 
[4]Susovan Jana; Matangini Chattopadhyay,An event-driven university campus navigation system on android platform,Applications and Innovations in Mobile Computing (AIMoC), 2015

[5]Mihaela Cardei; Iana Zankina; Ionut Cardei; Daniel Raviv, Campus assistant application on an Android platform, Southeastcon, 2013 Proceedings of IEEE

\section{ACKNOWLEDGEMENTS}

This work was financially supported by National Nature Science Foundation (61203152), Program for Liaoning Excellent Talents in University (LNET) (LJQ2013122),Natural Science Foundation of Liaoning Province (Grant No. 2015020037), General Project of the Education Department of Liaoning Province ( L2014473) and Program for graduate innovation and Entrepreneurship of Shenyang University(sdycxz2015032) 